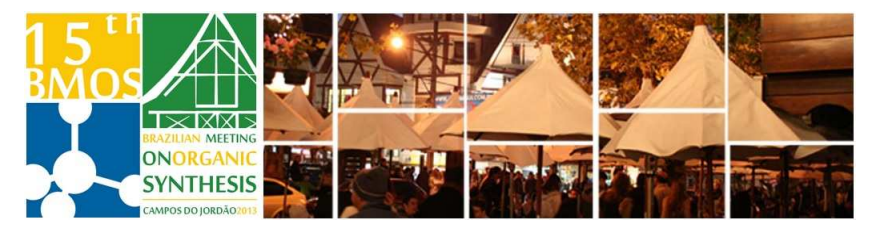

\title{
Base/Electrophile-Controlled Regioselective Functionalization of 1-Substituted-Indolizines
}

\author{
Mônica F. Z. J. Amaral*, Amanda A. Baumgartner, Giuliano C. Clososki \\ Research Center for Natural and Synthetic Products -Faculty of Pharmaceutical Sciences- University of São \\ Paulo, Ribeirão Preto, SP-Brazil \\ *monicaufg@gmail.com
}

Keywords: indolizine, directed metalation, organometallics

\section{INTRODUCTION}

The pyridine ring is present in a large amount of important polycyclic compounds. Among these, the indolizines stand out for being cores commonly found in nature (particularly in indolizidine alkaloids), in structure of substances of biological interest and for being the indole isosteres. ${ }^{1}$

Because of its importance, the optimization of the existing preparation methods or new development to facilitate the construction of indolizines functionalized is of great interest. Thus, the main objective of this project is to develop a regioselective methodology for the direct metalation of functionalized indolizines using organometallic bases $^{2}$ that can tolerate the presence of functional groups on the substrates.

\section{RESULTS AND DISCUSSION}

The organometallic bases were synthesized according to the procedures described in the literature. ${ }^{2}$ At the same time, indolizines of type 6 were synthesized through a 1,3-dipolar cycloaddition in the presence of a mild oxidant (Scheme 1).

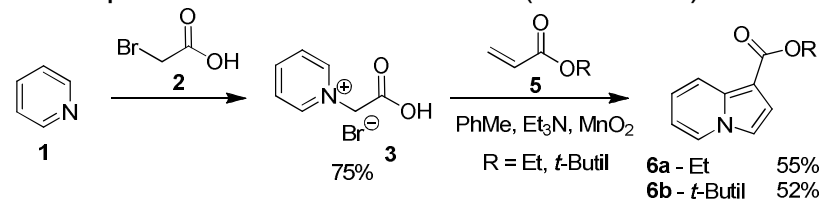

Scheme 1. Preparation of indolizines

First, we conducted a survey of reaction conditions for regioselective metalation. LDA, LiTMP, LiHMDS, DMPMgCl.LiCl and TMPMgCl.LiCl were used as base in different conditions (reaction temperature, reaction time and base equiv.). We have found that the best base for preparation of 5-substituted-1ester-indolizine was LDA in THF and for preparation of 2-substituted-1-ester-indolizine was TMPMgCl.LiCl in THF. Having established the conditions for efficient and regioselective metalation, we further investigated the reactivity of the organometallic intermediates with several electrophiles. The results are demonstrated in scheme 2 and figure 1. In general, LDA base produce indolizine 7 and $\mathrm{TMPMgCl}$. $\mathrm{LiCl}$ produce indolizine 8 in good regioselectivity. However, the use of the $\mathrm{TMSCl}$ as electrophile only gives the regioisomer $\mathbf{7}$, independently of base used.

Scheme 2. Selective direct metalation of $\mathbf{6 a}$ and $\mathbf{6 b}$ followed by reactions with Electropliles
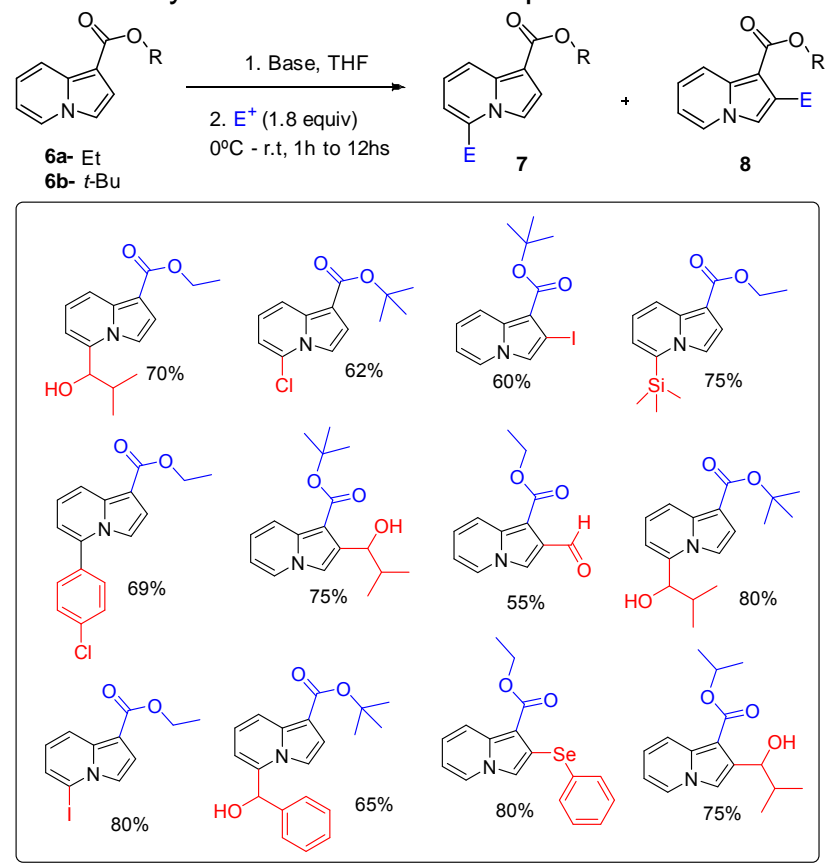

Figure 1

\section{CONCLUSION}

The direct metalation using organometallic bases appears to be an interesting method for the regioselective functionalization of indolizines. Additionaly, the use of the same protocol with different eletrophiles has allowed the preparation of a library of 2-substituted-1-ester-indolizine and 5substituted-1-ester-indolizine in moderate to good yields.

\section{ACKNOWLEDGEMENTS}

The authors are grateful for the support of FAPESP and CNPq for fellowship.

\section{REFERENCES}

1 a) Michael, J. P., Nat. Prod. Rep., 2001, 18, 520. b) Wang, Y. F.; Lu, C. H.; Lai, et al. Planta Med. 2003, 69, 1066-1068.

${ }^{2}$ Clososki, G. C.; Rohbogner, C. J.; Knochel, P. Angew Chem. Int. Ed., 2008, 47, 1503-1507. 